\title{
Cavernous sinus thrombosis
}

\author{
RE CLIFFORD-JONES, CJK ELLIS, JM STEVENS, A TURNER \\ From the National Hospital, Queen Square, London, UK
}

SUMMARY Four patients with clinical and investigative features suggestive of cavernous sinus thrombosis are reported. Radiological investigations included computed tomography of head and orbits. The problem of clinical and radiological distinction from orbital infection is discussed. Serious intracranial complications developed in two patients and the value of computed tomography in detecting these is stressed.

Cavernous sinus thrombosis was first reported as a pathological finding by Duncan in $1821 .^{1}$ Ten years later $\mathrm{Bright}^{2}$ gave the earliest clinical account but failed to mention the ocular abnormalities now considered so characteristic of the condition. These were described by Knapp in $1868^{3}$ and detailed by Eagleton ${ }^{4}$ in a personal series of 25 cases. Before the introduction of sulphonamides and penicillin Grove ${ }^{5}$ was able to collect 400 cases and noted that the mortality was practically $100 \%$. Yarrington ${ }^{6}$ reviewed the fall in mortality, since the introduction of antibiotics, to the current rate of $12-14 \%$. Cases are now reported less frequently but debate about the mechanism of symptom production continues and the impact of computed tomography (CT) on diagnosis and management has not yet been defined. We report our experience with four patients, whose investigations included CT of the head and orbits.

\section{Case reports}

Case 1, MF, B14259

A 58-year-old right handed female patient gave a three week history of an upper respiratory tract infection with pain in the face and intermittent headache. One week before admission the headache had become severe with nausea and vomiting. Five days later she was noted to be confused and unable to understand speech. She developed swelling and redness around the left eye which by the time of admission had spread to involve the right eye. She had a previous history of chronic sinusitis but had otherwise been well and had not received antibiotics prior to her admission. On examination she was febrile $\left(37 \cdot 2^{\circ} \mathrm{C}\right)$ with a mild dysphasia. There was prominent neck stiffness with a positive Kernig's sign. The maxillary and frontal bones were tender and there was extensive overlying swelling and erythema with pus in the nose. The conjunctivae were markedly oedematous and prolapsed through the

Address for reprint requests: Dr RE Clifford-Jones, The National Hospital, Queen Square, London WC1N 3BG, UK

Received 20 May 1982. Accepted 6 July 1982 palpebral fissure (fig 1). There was tense bilateral proptosis more marked in the left eye with firm resistance to retropulsion. Visual acuity could not be assessed accurately but she could fix and follow a small object. The visual fields were full and there was mild bilateral disc oedema without retinal venous engorgement. Movement of the left eye was limited to about $10^{\circ}$ in all directions and of the right eye there was similar limitation but for abduction which was present to $40^{\circ}$. The pupils were normal. There was a mild right hemiparesis with bilateral extensor plantar responses. Sensation was normal. General medical assessment showed a tachycardia. Initial investigations showed an ESR of $100 \mathrm{~mm}$ and a peripheral blood neutrophil leucocytosis. The cerebrospinal fluid was turbid, straw coloured and at a pressure of $310 \mathrm{~mm}$ of CSF. There were 350 white cells $/ \mathrm{cmm}$ (87\% polymorphs) with protein $1.84 \mathrm{~g} / \mathrm{l}$ and sugar 2.8 $\mathrm{mmol} / \mathrm{l}$ (45\% of blood sugar). No organisms were seen or cultured. One blood culture bottle grew anaerobic streptococcus. Plain skull radiographs showed opaque paranasal sinuses and CT scan showed a prominent scleral coat of each eye but no other abnormality.

Treatment was initiated with parenteral benzyl penicillin $12 \mathrm{~g} /$ day, flucloxacillin $2 \mathrm{~g}$ daily, gentamicin $240 \mathrm{mg}$ daily, metronidazole $1.5 \mathrm{~g} /$ day and dexamethasone $16 \mathrm{mg} /$ day. The patient's condition started to improve by the fourth hospital day, and she became afebrile on the eighth with diminishing ocular abnormality. The maxillary sinuses were drained on the fourth day of admission. During the first week the dysphasia and the mild right hemiparesis became more marked and a CT scan showed a subdural empyema over the left temporal convexity with some enhancement of the underlying frontal and temporal opercula. She continued to improve on antibiotic treatment which was maintained for one month. The dysphasia and hemiparesis had resolved by this time. By discharge the eyes were normal. Fourteen weeks after her admission the patient had a major seizure and she was started on phenytoin.

Case 2, AO, B15985

A 39-year-old woman had undergone a right transantral ethmoidectomy for pansinusitis. Six weeks later she developed severe headaches and within three days swelling around both eyes was noted. The patient then became confused and was admitted to hospital ten days after the onset 


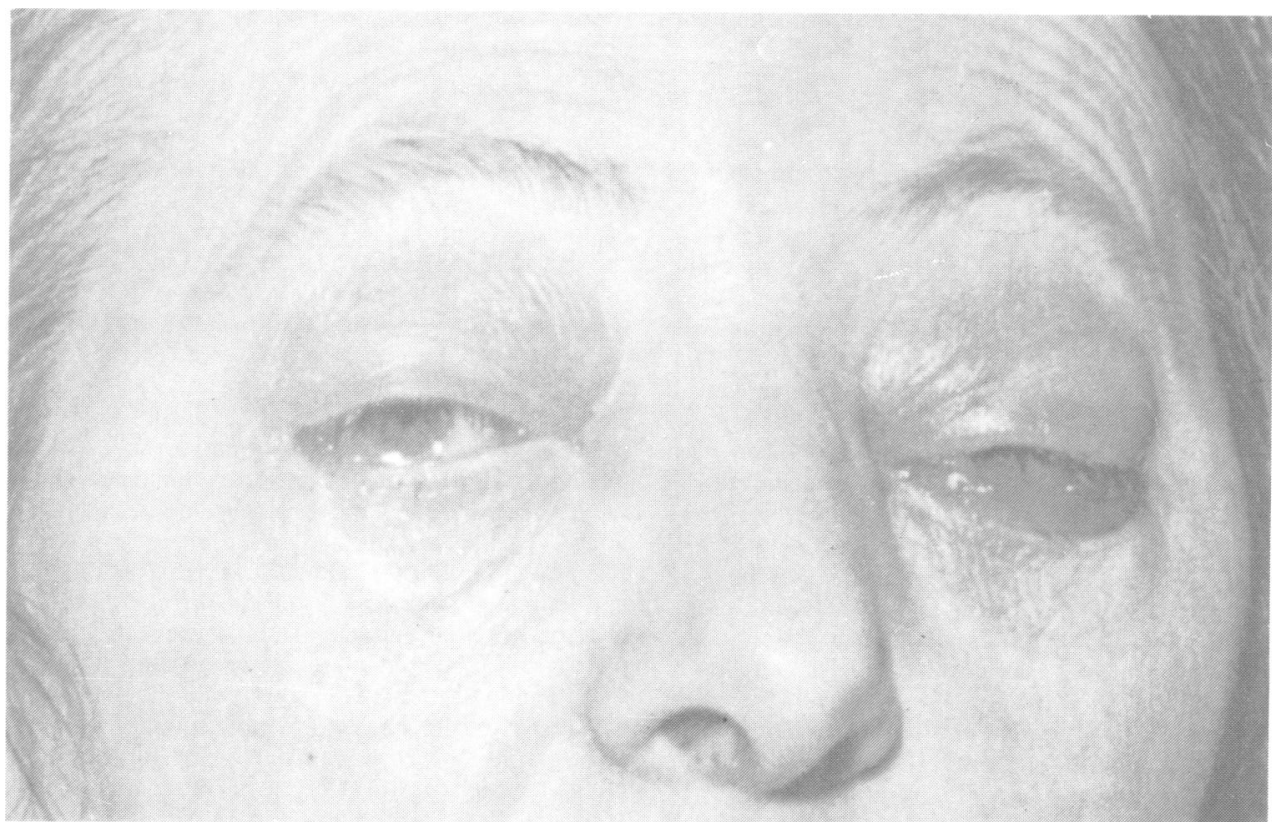

Fig 1 (Case 1) Facial appearance on admission. There is bilateral periorbital swelling. The conjunctivae are oedematous and prolapse through the palpebral fissures.

of her headaches. On examination, her temperature was $39^{\circ} \mathrm{C}$; she had a stiff neck and was very drowsy and disorientated. There was a tense bilateral axial proptosis with gross chemosis and some subconjunctival haemorrhages. The eyelids were swollen and discoloured. Visual acuity was reduced to counting fingers bilaterally and there was a left

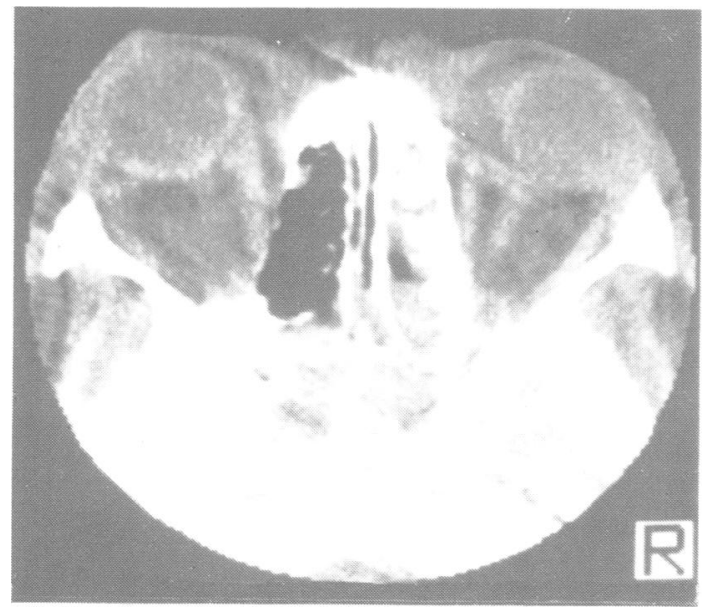

Fig 2 (Case 2) Unenhanced orbital CT scan. There is bilateral scleral thickening and preseptal swelling. The medial and lateral rectus muscles of the left orbit are thickened. Multiple retrobulbar opacities can be seen both inside and outside the muscle cone. homonymous hemianopia. The retinal veins in both fundi were dilated and the right disc showed early swelling. There was a complete bilateral external ophthalmoplegia with ptosis. The pupils, however, were normal. Both corneal reflexes were depressed and there was a flaccid left hemiplegia with dense sensory loss. Plain radiographs of the skull showed opaque paranasal sinuses. Orbital CT showed mild scleral thickening of each globe and multiple intraconal and extraconal retrobulbar opacities, which were either round or elongated. The medial and lateral rectus muscles were thickened in the left orbit and swelling in front of the orbital septum (preseptal swelling) was visible bilaterally (fig 2). Both cavernous sinuses appeared enlarged with convex lateral margins. Although CT of the head was normal initially, subsequent examinations showed the development of a large infarct deep in the right hemisphere. There was a marked neutrophil leucocytosis in the blood. The cerebrospinal fluid was turbid and yellow; the pressure was over $400 \mathrm{~mm}$ of CSF. It contained 1340 white cells/cmm ( $90 \%$ polymorphs); the protein was $1.05 \mathrm{~g} / \mathrm{l}$ and the sugar $4.4 \mathrm{mmol} / 1$ (47\% of blood sugar). No organisms were seen in the CSF and cultures were sterile. Similarly no organisms were isolated from the blood or from swabs of the ears, eyes, nose or throat.

The patient was treated with parenteral benzyl penicillin (12 g/day), metronidazole ( $1.5 \mathrm{~g} /$ day), flucloxacillin (4 $\mathrm{g} /$ day), gentamicin $(240 \mathrm{mg} /$ day) and dexamethasone (16 $\mathrm{mg}$ /day). Her general condition began to improve on the third hospital day; she was apyrexial on the seventh day and by the tenth day there was almost complete resolution of the chemosis and proptosis. Movements of the right eye returned , to normal but abduction remained restricted in the left eye. 
The hemiparesis improved over the next month and the patient was able to walk with the aid of a stick.

\section{Case 3, DP, MV 94881}

A 71-year-old woman had been aware of a hard lump over her right eye for several years. Over the five months before admission she had occasional diplopia and three weeks prior to entering hospital she developed cellulitis of her right leg. Her right eye became swollen three days before being admitted. On the evening of admission she became acutely confused and lapsed into a coma. On examination, her temperature was $38.5^{\circ} \mathrm{C}$; there was cellulitis of the right leg and marked neck stiffness. She was unconscious but moved all four limbs spontaneously. Focal fits involving the left arm and leg were observed. The right eye was proptosed and also deviated downwards in relation to a bony mass which could be palpated in the upper nasal part of the right orbit. There was gross chemosis of the right eye, with confluent subconjunctival haemorrhages and extensive swelling of upper and lower lids. The patient's visual acuity and fields could not be assessed on admission. The right disc was moderately swollen and the retinal veins dilated in both fundi. The right pupil was $3 \mathrm{~mm}$ in diameter and the left $5 \mathrm{~mm}$ but both responded normally to light and dark and there was no afferent defect. Oculocephalic testing revealed a complete external ophthalmoplegia of the right eye and normal eye movements on the left. The right corneal reflex was absent. There was a mild transient left hemiparesis and both plantars were extensor. The day after admission the patient's previously normal left eye became proptosed with swelling of the eyelids, chemosis and impaired abduction and elevation. Plain radiographs of the skull showed a large osteoma occupying most of the right frontal sinus. CT of the head and orbits showed no striking additional abnormality, but the scleral coats of each globe were prominent. There was a marked neutrophil leucocytosis in the peripheral blood. A lumbar puncture revealed turbid, yellow CSF under a pressure of over $400 \mathrm{~mm}$. The CSF contained 12330 white cells $/ \mathrm{cmm}$, all polymorphs, $3.2 \mathrm{~g} / 1$ of protein and $3 \cdot 1$ $\mathrm{mmol} / 1$ of sugar (36\% of bloud sugar). Microscopy and cultures of the fluid showed no organisms. Similarly no pathogens were isolated from blood cultures or swabs of eyes, ears, nose and throat. A CSF leak was identified in the posterior ethmoid regions by the use of small pieces of Dextrostix.

The patient was treated with intravenous benzyl penicillin (14.4 g/day), flucloxacillin (4 g/day), gentamicin (300 $\mathrm{mg} /$ day), metronidazole ( $1.5 \mathrm{~g} /$ day), dexamethasone (16 $\mathrm{mg} /$ day) and phenytoin ( $300 \mathrm{mg} /$ day). Her condition started to improve by the third hospital day. After a week the proptosis and ophthalmoplegia had improved and the chemosis, subconjunctival haemorrhages and fundal changes had all resolved. Some proptosis and restriction of movement of the right eye persisted due to the mechanical effect of the osteoma, but by discharge at six weeks the rest of her neurological deficit had resolved.

Case 4, $M V, 96044$

A 24-year-old male patient gave a two month history of cough, intermittent headaches and nasal discharge. Four weeks before admission he developed severe headache which resolved over two days, but recurred a week prior to entering hospital. On this occasion the headache was associated with drowsiness, photophobia and vertical diplopia worse on looking to the right. On examination, the patient was pyrexial $\left(39^{\circ} \mathrm{C}\right)$ with marked neck stiffnes. He was drowsy and disorientated. The right eye was proptosed with swelling of the eyelids and erythema over the right side of the face. The conjunctiva of the right eye was oedematous. Acuity was J18 on the right and $\mathrm{J} 1$ on the left. The visual fields and fundi showed no abnormality. There was a marked ptosis of the right eye, which the patient could only just open. The pupils were equal, $2 \mathrm{~mm}$ in size and reacted normally. A marked external ophthalmoplegia was observed on the right side with abduction and elevation of the eye being severely restricted. There was mild weakness of abduction of the left eye. The right corneal reflex was depressed and sensation was impaired in all three divisions of the right trigeminal nerve. Trismus of the jaw was present. No neurological deficit was noted in the limbs. Two days after admission the left eye became chemotic. Plain radiographs of the skull showed opacity of the sphenoid and ethmoid sinuses. CT of the orbits showed bilateral preseptal swelling and scleral thickening of the globes, greater on the right. The right lateral rectus muscle was thickened and there was an ill-defined density behind the right globe (fig 3). CT scans of the head were normal but bilateral carotid angiography showed smooth narrowing of the intracavernous portion of each carotid artery, again more marked on the right (fig 4). The peripheral blood showed a neutrophil leucocytosis. The spinal fluid was opalescent and under increased pressure $(280 \mathrm{~mm}$ of CSF) and contained 320 white cells/cmm ( $92 \%$ polymorphs). The CSF protein was $0.98 \mathrm{~g} / \mathrm{l}$ and the sugar $4.4 \mathrm{mmol} / \mathrm{l}$ ( $57 \%$ of blood sugar). Microscopy and culture of the fluid revealed no organisms but an anaerobic streptococcus was grown from one blood culture bottle.

The patient was initially treated with parenteral benzyl penicillin (16 megaunits/day), cefuroxime ( $6 \mathrm{gm} / \mathrm{day})$ and metronidazole ( $1.5 \mathrm{gm} /$ day). The pyrexia, meningitic signs and orbital abnormalities resolved almost completely over four days. The maxillary and ethmoid sinuses were drained on the twentieth hospital day. By discharge after one month in hospital the patient had some residual subjective sensory

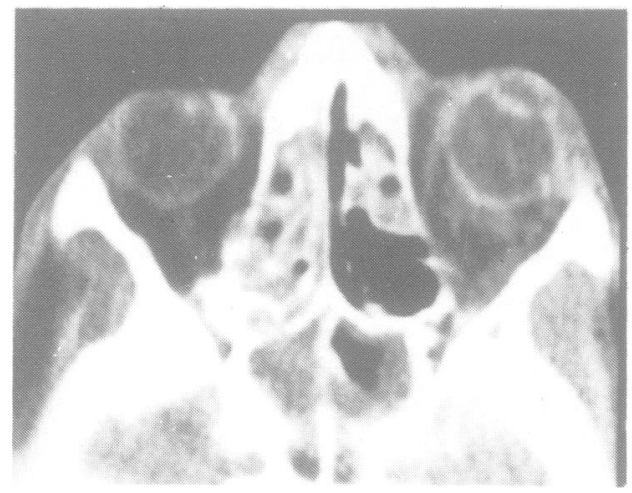

Fig 3 (Case 4) Unenhanced orbital CT scan showing scleral thickening greater on the right. The right lateral rectus muscle is thickened and there is an ill defined density behind the right globe. 

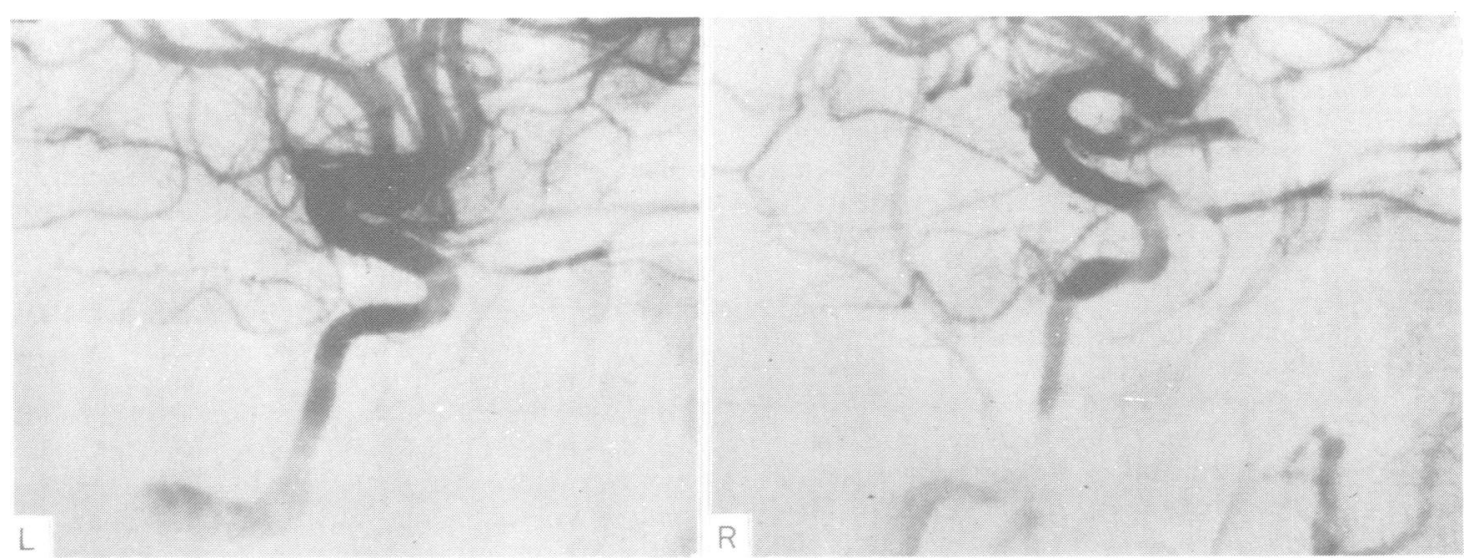

Fig 4 (Case 4) Left and right carotid angiograms in lateral projection showing diffuse narrowing of the extradural and intracavernous carotid arteries, more marked on the right side.

change over the right side of the face and mild weakness of abduction of the left eye.

\section{Discussion}

The clinical presentations of our patients are similar to those of previously reported cases of cavernous sinus thrombosis. ${ }^{4-9}$ Disease of the paranasal sinuses was present in all four cases and was the presumptive cause for the development of cavernous sinus thrombosis. In three there was sinusitis, one had also had a recent sinus operation and the fourth was found to have a cerebrospinal fluid (CSF) leak in relation to a frontal osteoma. In a previous review ${ }^{8}$ the primary focus of infection was found more commonly to be facial furuncles than sinusitis.

A short history of severe headache, marked toxaemia and clouding of consciousness, with orbital swelling was noted in each patient. Double vision has been described ${ }^{10}$ as a premonitory symptom of cavernous sinus thrombosis but was an early symptom in only one of our patients. By the time of admission the patients had a marked systemic illness with signs of meningitis. In all cases there were striking orbital abnormalities with proptosis, facial erythema, lid swelling and chemosis. In three patients the orbital changes were initially unilateral with subsequent and less marked involvement of the other side. Bilateral abnormalities were present from the onset in case 2 . Extensive subconjunctival haemorrhages occurred in two patients. Eye movements were restricted apparently in proportion to the degree of the other orbital signs on the corresponding side. Mild orbital swelling was associated predominantly with weakness of abduction and elevation of the eye, whereas a complete external ophthalmoplegia was observed with severe orbital involvement as evidenced by tense axial proptosis, conjunctival oedema and haemorrhages. By comparison with these striking orbital and external ocular movement abnormalities the optic nerves and pupils were relatively unaffected. Visual fields and acuities were usually normal by the time they could be assessed accurately, and the optic discs were either normal or only mildly swollen with some retinal vein dilatation but no haemorrhages.

There has been debate about the mechanism of the restriction of ocular movements in cavernous sinus thrombosis. Both muscle swelling as a result of the orbital oedema and neural damage in the cavernous sinus could be involved and indeed Walsh ${ }^{11}$ has shown that there was histological evidence of orbital muscle infection in all of six cases which came to necropsy. Aberrant regeneration of the third nerve has been reported $^{12}$ during recovery from cavernous sinus thrombosis and in this case demonstrated unequivocal evidence of neural involvement. However, the following features of the ophthalmoplegia in our cases suggested that muscle swelling was its predominant cause. The extent of the ophthalmoplegia appeared to be related to the degree of orbital abnormality at various stages of the patients' illnesses. Both ophthalmoplegia and the other orbital signs resolved rapidly with treatment. The pupils were often normal at a stage when there was a total external ophthalmoplegia. Other neurological deficits common in our patients were depression of the corneal reflex and weakness of the limbs contralateral to the worse affected eye.

The CSF was discoloured and under increased pressure in all cases. Examination of the CSF revealed a characteristic pattern of large numbers of white cells, almost exclusively polymorphs, a raised protein level 
and a slightly low sugar relative to the blood level. All CSF parameters were most severely disturbed in case 3 whose conscious level was most severely impaired. Cultures of the CSF in the four cases were uniformly sterile but anaerobic streptococci were isolated from the blood of two patients. The surprising infrequency of positive CSF cultures, and therefore the importance of taking blood cultures, has been reviewed by Taylor. ${ }^{13}$

Radiological investigations showed paranasal sinus disease in all cases, and CT of the head revealed intracranial complications in two. In cases 1 and 3 orbital CT showed only slight scleral prominence, though the images were of poor quality; normal orbital appearances at CT have been reported with cavernous sinus thrombosis. ${ }^{14}$ In cases 2 and 4 definite orbital abnormalities were seen and included preseptal swelling, swelling of extraocular muscles, scleral thickening, and retrobulbar densities. These findings have also been seen in acute orbital inflammation. ${ }^{15-17}$

Case 4, clinically the mildest affected, showed narrowing of both carotid syphons on angiography and case 2 probably suffered an internal carotid thrombosis. Both are familiar observations in cavernous sinus disease. ${ }^{14}{ }^{18}$ Abnormalities of the cavernous sinuses have been noted at carotid angiography, but venography via the orbital veins, or better, via the inferior petrosal sinus has been described ${ }^{14} 1920$ as the most appropriate contrast examination to demonstrate the veins in this region. However, venography performed via an infected orbit carries a risk of intracranial dissemination of infection or may even induce extension of thrombosis, regardless of the route by which it is injected. ${ }^{21} 22$

CT of the orbits in case 2 showed a feature of particular interest not previously reported. Well defined retrobulbar opacities which were either rounded or elongated were distributed throughout the intraconal and extraconal spaces. The pattern was unlike that seen in orbital infection and probably represented orbital veins. These have also been seen in carotid-cavernous sinus fistula ${ }^{23}$ but then it was usually the superior ophthalmic vein which was predominantly or exclusively involved. Scans of this patient also showed enlarged cavernous sinuses but it was the only case where the sinuses were clearly visible. The cavernous sinuses and their nonvascular contents may well be seen in axial and coronal CT using high resolution and slice thickness of $3 \mathrm{~mm}$ or less. ${ }^{24}$ Contrast enhanced CT has permitted visualisation of thrombus in the superior sagittal sinus ${ }^{25}$ and sophisticated CT equipment should be able to document thrombosis of the cavernous sinuses. In our cases, the available slice thicknesses of 5 or $10 \mathrm{~mm}$ did not allow sinus contents to be distinguished from enhancing dura and adjacent bone.
Wide spectrum high dose antibiotic treatment was started in these critically ill patients immediately after their initial investigations and in the absence of bacteriological identification of the responsible organism. Yarrington ${ }^{6}$ has attributed the dramatic fall in the mortality of cavernous sinus thrombosis in the last 20 years to such aggressive therapy. The addition of steroids has both theoretical ${ }^{12}$ and clinical ${ }^{26}$ support and was instituted in all but the mildest affected of our patients. The role of anticoagulants remains controversial $^{27}$ and no patient in this study received them. Although the course of the illness in each patient was protracted, all patients started to improve within a few days of starting treatment and the orbital signs and ophthalmoplegia had largely resolved within a week. Two patients developed serious intracranial complications; in case 3 a deep capsular infarct led to a persistent hemiparesis and in case 1 a subdural empyema resulted in temporary worsening of the patient's neurological deficit and later in epilepsy. However, by discharge from hospital, all patients were independent although mild residual defects of cranial nerve function were common as stressed by Shaw. ${ }^{8}$

In conclusion, the diagnosis of cavernous sinus thrombosis remains essentially a clinical one and was based in our cases on their close resemblance to previous pathologically confirmed reports. In addition two cases had radiographic evidence of abnormality in relation to the cavernous sinus. The most difficult clinical distinction is from orbital cellulitis as proptosis, chemosis and periorbital inflammation may be marked in both conditions. ${ }^{16}$ Bilateral involvement and signs of CNS infection are important features distinguishing cavernous sinus thrombosis from orbital cellulitis. ${ }^{6-9}$ However, the pathological studies of Walsh ${ }^{11}$ suggest that, whereas orbital cellulitis may be an isolated phenomenon, in cavernous sinus thrombosis infection of the orbital tissues usually coexists with sinus thrombosis. Both processes probably contribute to the physical signs in this condition. CT scanning can reveal the associated orbital changes and is important in detecting intracranial complications which may develop and impair recovery. Angiography or venography can provide confirmatory evidence of cavernous sinus involvement but are potentially hazardous and should not delay the vigorous treatment necessary in these patients. In centres where the later generations of CT scanners are available, CT should perhaps be the only imaging test necessary to document the clinical diagnosis of cavernous sinus thrombosis.

We thank Professsor RW Gilliatt, Dr RS Kocen, Dr JA Morgan-Hughes and Dr P Rudge for permission to report these cases and to Miss Ann Woolley for typing the manuscript. 


\section{References}

1 Duncan A. Contributions to Morbid Anatomy. Edin Med Surg J 1821;17:321-36.

2 Bright R. Reports of Medical Cases. London: Longman, 1831. Vol 2. 129-32.

${ }^{3}$ Knapp H. Ueber Verstopfung der Blutgefässe des Auges. Albrecht Von Graefes Arch Klin Exp Ophthalmol 1868;18:207-51.

${ }^{4}$ Eagleton WP. Cavernous Sinus Thrombophlebitis. New York: Macmillan, 1926.

5 Grove WE. Septic and aseptic types of thrombosis of the cavernous sinus. Arch Otolaryngol 1936;24:29-50.

6 Yarrington CT. Cavernous sinus thrombosis revisited. Proc R Soc Med 1977;70:456-9.

7 Langworthy HG. Anatomic relations of the cavernous sinus to other structures, with consideration of various pathologic processes by which it may become involved. Ann Otol Rhinol Laryngol 1916;25:554-86.

* Shaw RE. Cavernous Sinus Thrombophlebitis: a review. Br J Surg 1952;40:40-8.

9 Walsh FB, Hoyt WF. Clinical Neuro-ophthalmology, Ed 3. Baltimore: Williams and Wilkins, 1969: 1892-4.

10 Pascarelli E, Lemlich A. Diplopia and photophobia as premonitory symptoms in cavernous sinus thrombosis. Ann Otol Rhinol Laryngol 1964;73:210-7.

1 Walsh FB. Ocular signs of thrombosis of the intracranial sinuses. Arch Ophthalmol 1937;17:46-65.

12 Brown P. Septic cavernous sinus thrombosis. Bull Johns Hopkins Hosp 1961;109:68-75.

13 Taylor PJ. Cavernous sinus thrombophlebitis. $\mathrm{Br} \mathrm{J}$ Ophthalmol 1957; 41:228-37

14 Sekhar LN, Dujouny M, Rao GR. Carotid-cavernous sinus thrombosis caused by Aspergillus fumigatus. $J$ Neurosurg 1980;52:120-5.

15 Leo JS, Halpern J, Sackler JP. CT in the evaluation of orbital infection. Comput Tomogr 1980;4:133-8.

16 Zimmerman RA, Bilanick LT. CT of orbital infections and its cerebral complications. Ann J Rc diol 1980:134: 45-50.

17 Nugent RA, Rootman J, Robertson WD, Lapointe JS, Harrison PB. Acute orbital pseudotumours: classification and CT features. Am J Neuroradial 1981;2: 431-6.

18 Brismar G, Brismar J. Thrombosis of the intraorbital veins and cavernous sinus. Acta Radiol (Diagn) (Stockh) 1977;18:145-53.

19 Doyon DL, Aron-Rosa DS, Ramée A. Orbital veins and cavernous sinus. In: Newton TH, Potts DG, eds. Radiology of Skull and Spine Book 3. St Louis: Mosby 1974, 2220-56.

20 Yasargil GM, Damur M. Thrombosis of cerebral veins and dural sinuses. In: Newton TH, Potts DG, eds. Radiology of Skull and Spine Book 4. St Louis: Mosby 1974, 2375-400

21 Ritchie WGM, Lynch PR, Stewart GJ. The effect of contrast media on normal and inflamed canine veins. A scanning and transmission electron microscopic study. Invest Radiol 1974;9:444-55

22 Seeger JF, Gabrielson TO, Giannotta SL, Lotz PR. Carotid-cavernous sinus fistulas and venous thrombosis. Ann J Neuroradial 1980; 1:141-8.

23 Merrick R, Latchaw RE, Gold LHA. CT of the orbit in carotid-cavernous sinus fistulae. Comput Tomogr $1980 ; 4: 127-32$

24 Kline LB, Acker JD, Donovan Post MJ, Vitek JJ. The cavernous sinus: a computed tomographic study. Ann J Neuroradial 1981;2:299-305.

25 Buonanno FS, Moody DM, Ball MR, Wayne Laster D. Computed cranial tomographic findings in cerebral sinovenous occlusion. J Comput Assist Tomogr 1978; 2:281-90.

${ }^{26}$ Soloman OD, Moses L, Volk H. Steroid therapy in cavernous sinus thrombosis. Am J Ophthalmol 1962; 54:1122-5.

27 Parsons M. Intracranial venous thrombosis. Postgrad Med $J$ 1967;43:409-14. 